\title{
Muerte digna o ensañamiento terapéutico: una reflexión para profesionales de la salud
}

Mtra. Teresa de Jesús Salazar Gómez*, EEAEC. Méndez Xavier Gabriela**, Mtra. Gandhy Ponce Gomez***, Lic. Claudia María Garduño Ortega**** * Profesor de carrera asociado"C" de la ENEO-UNAM, ** Estudiante de la Especialidad de Adulto en Estudio Crítico,

*** Profesor de carrera asociado "B", ENEO-UNAM, **** Profesoe de asignatura "A", ENEO-UNAM.

El acompañamiento durante el sufrimiento humano se vuelve un desafío a la reflexión bioética, debido a una avanzada edad, a la gravedad de la enfermedad, a la condición terminal y al abandono familiar de pacientes en estas situaciones, y por tanto al dilema de la utilidad de algunos procedimientos y la consecuente actitud del profesional de enfermería. Centro de estos problemas es la concientización de los cuidados de enfermería y la relación enfermera-paciente en una situación de enfermedad terminal (SET)

Se trata de un Estudio de caso producto de la práctica de los alumnos de la especialidad de post grado en Enfermería del Adulto en estado Crítico aplicando el Proceso de Atención de Enfermería basado en las 14 necesidades de Virginia Henderson realizado a una persona portadora de Cor Pulmonale. Su objetivo es analizar la evolución clínica de la información registrada siguiendo la metodología de estudio de casos. El marco teórico y el posterior análisis está centrado en la utilización de los conceptos ensañamiento terapéutico y rol de enfermería en el marco que proporciona la reflexión bioética contemporánea sobre el derecho a una muerte digna.

Palabras clave: Enfermería / cuidados intensivos / bioética / ensañamiento terapéutico/ ortotanasia / distanasia

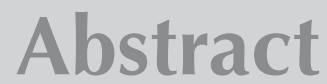

The company or accompany during human suffering becomes a challenge to bioethics reflection, due a very old age, the illness severity, a terminal condition, and the family abandon of patients in these situations, and therefore, also for the dilemma of the usefulness of some procedures and the consequent attitude of the nursing professional. The core of these problems is the conscious of nursing care and the nurse-patient relationship within a terminal illness situation (SET).

This is a case study product of the practice of nursing specialized on critical state adults students, applying the Nursing Attention Process based on the Virginia Henderson's 14 needs on a person carrying the Cor Pulmonale. The object to be analyzed is the clinical evolution of information registered following the case study methodology.

The theoretical frame and later analysis is centered on the use of therapeutically enragement concepts and the nursing role in the frame that gives the contemporaneous bioethical reflection about the right to a honorable death.

Key word. Nursing/ intensive care/ bioethics/ therapeutically enragement/ ortotanasia / distance.

\section{INTRODUCCIÓN}

El ensañamiento terapéutico se ha transformado en un problema ético de primer orden para las ciencias de la salud, una vez que un conjunto de procesos biológicos que tenían alta probabilidad de muerte, pasaron a ser pasibles de control mediante los avances tecnológico-médicos. El objetivo es analizar la evolución clínica de un estudio de caso utilizando los constructos: ensañamiento terapéutico y el rol de enfermería en el marco que proporciona la reflexión bioética contemporánea sobre el derecho a una muerte digna. La reflexión parte de la convivencia y cuidado de una anciana en situación de enfermedad terminal (SET), entendida como la circunstancia en la que una o varias enfermedades no curables (bien porque no han respondido al tratamiento, bien porque no existen tratamientos curativos): a) amenazan la vida del enfermo en un plazo de meses (6 meses es la cifra más aceptada); b) 
producen síntomas cambiantes que afectan severamente la capacidad funcional, el estado mental y emocional del paciente; y c) éstos síntomas producen un gran impacto en la familia o allegados'.

El acompañamiento durante el sufrimiento humano se vuelve desafío a la reflexión bioética, debido a una avanzada edad, a la gravedad de la enfermedad, a la condición terminal, y por tanto al dilema de la utilidad de algunos procedimientos, la actitud del profesional de enfermería y al abandono familiar de personas en estas situaciones. En el centro de estos problemas está el sentido que se debe de dar a los cuidados de enfermería y a la relación enfermerapaciente en una situación de enfermedad terminal (SET).

Hoy en día, cuando los valores están cambiando en los individuos, donde la ecuación costo/beneficio parece primar en la elección de un tratamiento, cuando la ciencia y la técnica marcan prácticamente todos los espacios y ritmos de la atención de enfermos críticos, se corre el riesgo de perder la sensibilidad humana, y de sentir afecto por la persona que está sufriendo. La máquina podrá monitorear los signos vitales de la persona con total precisión pero no podrá aliviar su angustia, acompañar el dolor, conversar sobre el sentido de lo que se está viviendo o proporcionar un ambiente más humano en los últimos momentos de la vida.

\section{Sufrimiento y dolor.}

Al respecto del sufrimiento humano, se requiere de iniciar una vinculación conceptual entre el dolor y el sufrimiento, que pocas veces son directrices en el cuidado a personas en SET. Al respecto se hace referencia que desde 1979 la Asociación Internacional del Estudio del Dolor definió a este como una experiencia emocional y sensorial desagradable asociada con el daño potencial o actual de tejidos descrita en términos de tales cambios². La inglesa Cicelly Saunders acuñó la expresión dolor total, que incluye además del dolor físico, las nociones de dolor mental, social y espiritual ${ }^{3}$. El sufrimiento ha sido definido como una experiencia de impotencia causado por un dolor no aliviado; una situación de enfermedad prolongada que lleva a interpretar la vida como vacía de sentido ${ }^{4}$. En consecuencia, el sufrimiento sería de acuerdo con la conceptualización descrita, más global y permanente que el dolor, y en esencia sería sinónimo de calidad de vida disminuida.

Aunque el dolor físico sea la fuente más común de sufrimiento, el dolor en el proceso de morir va más allá de lo físico y debiera ser comprendido y atendido en sus dimensiones psíquicas, sociales y espirituales. Esta distinción entre curar / cuidar y entre dolor y sufrimiento, está en la base, por ejemplo, del modelo de atención de Saunders (1980) y de la creación de los "Hospice". También permite pensar un paradigma del cuidado distinto al paradigma del curar.

\section{Situacion de enfermedad terminal}

El constructo de "paciente terminal" se constituye de la unión de los vocablos: paciente y terminal. Al paciente por etimología se le como quien debe tener paciencia en la cura, y se le confiere la característica de mantener una actitud de espera pasiva, la que será satisfecha por el saber médico y/o por la tecnología disponible.

Independientemente, el vocablo "terminal" es sinónimo habitual de: "desahuciado", "agonizante", "gravemente enfermo", "moribundo", "incurable", entre otros, que etiquetan tanto a enfermos críticos como a graves u otros cuya patología excede dichos encuadres clínicos.

Haciendo una inferencia de ambos vocablos, se tiene una visión del "paciente terminal", como aquella persona rotulada como tal, en una posición de inferioridad, en la que su esfera decisoria se encuentra restringida a su mínima expresión, o directamente anulada.

\section{Muerte digna}

La "muerte digna", también denominada "ortotanasia" implica dos constructos, que lo son por la complejidad de su definición. Algunos autores sostienen una postura radical, el sentido profundo de la dignidad de morir surgirá inevitablemente al recuperarse la dignidad de la vida. En una persona en situación de enfermedad terminal, la muerte es su propia muerte. La dignidad viene de la apropiación que la persona logre de este momento:

"Nadie, ni siquiera un experto puede ni debe controlar la muerte de otro. Esta voluntad debe ser respetada aunque el enfermo ya no esté en condiciones de tomar decisiones. La incapacidad del enfermo, su inconciencia, nunca pueden servir de excusa o pretexto para ignorar su derecho a una muerte digna"5.

Esta posición reconoce radicalmente que para el enfermo la muerte es "su" muerte. Cuando está conciente es normal que reivindique el derecho a dirigir el proceso según sus expectativas, convicciones y valores propios. Una muerte no digna será aquella en la que una persona decide sobre la muerte del paciente; otros que impiden el morir, tratando de hacer "algo" cuando la muerte es ya inevitable. En consecuencia, sólo la propia persona agonizante puede definir sobre la dignidad de su muerte, nadie lo puede hacer en su lugar. 
Una segunda posición incluye otras consideraciones respecto de la dignidad de la muerte. Una muerte será calificable de digna cuando: a) se ha producido con todos los alivios médicos adecuados, disminuir el dolor sin que lleguen a ser desproporcionados y b) todos los consuelos humanos posibles. Una muerte digna es en esta posición, una muerte en lo posible conciente para la persona y "humanizada" para el equipo de salud y los allegados.

Dentro de los consuelos humanos, el equipo de salud debe proporcionar a la persona que agoniza la posibilidad de asumir su muerte, expresando sus sentimientos en el momento, reflexionando sobre su existencia, y promoviendo que cada uno según sus creencias religiosas, pueda vivir ese proceso en clave espiritual ${ }^{2}$. Por lo general, hay que reconocer que tanto los allegados como el equipo de salud no suelen favorecer este tipo de reflexión. Al contrario, en ocasiones se oculta la proximidad de la muerte; se trata de no hablar sobre ella con la persona porque se supone que esto tenderá a angustiarla más. Por "paternalismo" se evita hablar de este "mal".

\section{Ensañamiento terapeutico}

El ensañamiento terapéutico "distanasia" u "obstinación terapéutica" es un intento de retardar lo más posible la muerte, gracias a una intervención médica respecto a tres criterios objetivos en el marco de la bioética, que no dependen sólo del familiar o del médico: a) inutilidad, cuando se trata de una cura que resulta del todo ineficaz e inútil: "podemos continuar, pero incluso continuando no obtenemos resultados", por ejemplo cuando nos encontramos ante una situación de irreversibilidad, generalmente definida por la muerte cerebral, es verdaderamente inútil continuar; b) la gravosidad, o sea de la pena excesiva a la que estaría expuesto el enfermo, el cual terminaría por sufrir de más, ya sea físicamente o moralmente. Y c) excepcionalidad, o sea cuando se interviene con medios que son desproporcionados. Este es un criterio muy relativo, que cambia con el tiempo ${ }^{6}$. Cuando se verifican juntos estos tres criterios, nos encontramos ante el ensañamiento terapéutico $y$, desde el punto de vista moral, podemos renunciar, debemos renunciar o proseguir con el tratamiento. Aunque como profesionales de enfermería y equipo de la salud, hay un asunto particular al que se debe prestar atención: incluso cuando nos encontrásemos en esta situación, debemos continuar suministrando los cuidados ordinarios, como dar de beber y de comer. Sobre todo, no debe jamás faltar el cuidado humano fundamental, que es el de estar presentes y de compartir de algún modo el momento de la muerte.

\section{METODOLOGIA}

El estudio se realizó en un hospital de la ciudad de México, producto de las prácticas establecidas por el Programa de Post-grado en Enfermería de Adulto en Estado Crítico de la Escuela Nacional de Enfermería y Obstetricia de la Universidad Nacional Autónoma de México UNAM.

Tiempo: 15 de septiembre al 31 de octubre de 2003

Tipo de Estudio: Prospectivo y documental.

\section{Presentacion del caso}

Mujer de 81 años de edad, soltera, originaria de Michoacán, pero residente desde hacía 50 años en el Distrito Federal. Medía $165 \mathrm{~cm}$ y pesaba 90 kilos;. Un indicador de su origen socioeconómico bajo es que el máximo nivel de educación formal alcanzado correspondía $4^{\circ}$ año de Primaria.

Desde un punto de vista cultural, la señora estaba formada en una concepción y en valores arraigados respecto al pudor personal. Esto se hacía evidente en particular, en los momentos en que se realizaba la higiene corporal o algún otro procedimiento más invasivo de su intimidad.

En lo que respecta a la dimensión espiritual, profesaba la religión católica y se observaba entre sus pertenencias, varios símbolos de la expresión de su fe como un rosario e imágenes de la Virgen.

Se desconocían datos respecto a vivienda, alimentación y ocupaciones, ya que no se pudo interrogar con la profundidad y amplitud necesaria a la señora debido al deterioro de su estado de salud que se observó a partir del tercer día de ingreso.

Finalmente, durante el período de la internación sólo se hizo presente un familiar no cercano a la señora, esta persona al comienzo venía en la hora de la visita, le traía diferentes cosas y hablaba con ella, al cabo de una semana no concurrió más, no hubo familiares directos de la señora y tampoco los mencionó ella; en consecuencia sufrió abandono, y fue derivada a un asistente social del Hospital quien asumió la curatela.

\section{Antecedentes clínicos}

La mayoría de los datos sobre sus antecedentes fueron extraídos de su historia clínica, en la misma constata que la señora no era fumadora. Desde los 18 años (1940) padecía de Bronquitis Crónica, a causa de la cual desarrolló 
posteriormente Enfermedad Obstructiva Crónica (EPOC). A los 74 años (en 1996) se le diagnosticó Cor Pulmonale Crónico, de padecer Hipertensión Arterial Sistémica, ignorándose la fecha de diagnóstico.

La señora ingresó a la UCIN el día 22 de octubre de 2003, con diagnóstico de Cor Pulmonale descompensado, exacerbado con Neumonía Nosocomial. Procedía del Servicio de Neumología del mismo Hospital en donde había estado internada desde hacía 15 días.

\section{Evolución clínica y paraclínica}

A partir de su ingreso a la Unidad de Cuidados Intensivos Neurológicos (UCIN), su estado físico fue deteriorándose cada vez más en forma irreversible. De acuerdo con la valoración de las 14 necesidades la evaluación clínica y los detalles de su proceso patológico fueron los siguientes.

\section{Rol de Enfermerìa ante el caso.}

El equipo de Enfermería estaba integrado por la enfermera jefa de sala, tres enfermeras por turno y siete estudiantes del Post-grado de ENEO, para un total de siete enfermos.

Respecto a la necesidad de respirar debida a la insuficiencia respiratoria, requirió asistencia ventilatoria mecánica los 45 días que permaneció en la UCIN, trece días más después del ingreso, fue necesaria la realización de una traqueostomía. Fue imposible el destete del Apoyo Ventilatorio Mecánico (AVM) por un tiempo no mayor de 15 minutos, ademas presento alteraciones del sistema cardíaco, ya que a cuatro días del ingreso, manifesto una disminución del gasto cardíaco, así como extrasístoles ventriculares, por lo cual requirió la administración de inotrópicos así como digitálicos. Un factor exacerbante desde un comienzo es la anemia; luego plaquetopemia, leucocitosis y finalmente leucopenia producida por la sepsis.

Para suplir la necesidad de alimentación se instauró la nutrición enteral mediante sonda nasogástrica. Posteriormente se observa sangrado de tuvo digestivo alto y bajo, por lo cual debe realizarse lavado gástrico, reposición de volúmenes con hemoderivados y se instaura nutrición parenteral.

En la eliminación a nivel renal, a comienzos de noviembre se instaura una insuficiencia pre-renal aguda y anuria.

En la necesidad de protección de la piel se inició con la identificación de úlceras por presión, grado II en los talones y región sacrocoxigea, relacionado con el reposo prolongado y el edema generalizado. Aparece laceración en cuello ocasionado por la fijación de la traqueostomía y en muslo derecho por la fijación de la sonda vesical. Se observaron múltiples hematomas en miembros superiores debidos a punciones arteriales, realizadas por turno.

Los registros en los que se basó el estudio de caso, se realizaron en su totalidad por la estudiante de la especialidad durante el turno matutino. Se pudo constatar que en algunas ocasiones existía déficit de higiene y confort producto de la no satisfacción de estas necesidades durante el turno nocturno de enfermería y se le encuentra en 6 ocasiones con medidas de sujeción. En adelante sólo se hará referencia a lo observado y al desempeño de los tres roles de cuidado: informar, abogar por sus intereses y valores, y comprender empáticamente a la usuaria en su proceso vital.

La comunicación con la señora sobre su enfermedad y en particular sobre los procedimientos de enfermería que se le estaban realizando tuvo un cambio. Inicialmente, los primeros 10 días, cuando su estado de ánimo era más favorable y tenía una disposición a colaborar, la señora era informada sucintamente sobre cada procedimiento (higiene y confort, administración de medicamentos). En el caso de las gasometrías, éstas eran realizadas por el técnico de inhaloterapia de turno, quien en ocasiones, además de informarle, también bromeaba con ella.

Sin embargo, conforme su estado de ánimo iba decayendo fue necesario pasar del plano de la mera información a la negociación sobre los cuidados. Es decir, era necesario conversar con ella más extensamente para convencerla de los beneficios que obtendría luego de cada cuidado y así obtener su consentimiento para realizar cada práctica. La comunicación se amplío y diversificó no sólo en sus propósitos, sino en la duración, en la frecuencia y en los contenidos. Este rol de más activo involucramiento con la señora fue desempeñado principalmente por la estudiante.

Le realizarón un tacto vaginal en el que se argumentaba la necesidad de exploración por sangrado a ese nivel, a ese respecto se ejerció el rol de abogar por los valores de la señora. Frente a la decisión médica, se optó por exponer los valores de la persona sin hacer alusión a la poca relevancia de los datos que conseguirían, y la estudiante reaccionó rechazando la pertinencia ética de tal procedimiento.

Se le comentó al médico acerca de los temores y pudor así como la negación de vida sexual activa de la señora y que desde el punto de vista ético, la oscultación probablemente la expondría a una situación muy vergonzosa, 
la dejaría muy alterada y constituiría un menoscabo a su dignidad. Sin embargo, el médico no consideró la opinión profesional y procedió a realizar el tacto, sin éxito en el procedimiento puesto que la señora era núbil.

Fue de esperar que la comunicación y en general la relación de la señora con el equipo de enfermería, fue radicalmente transformada luego del episodio de la auscultación vaginal. La señora quedó muy alterada ese día, rechazando los cuidados. En días siguientes conservó una reacción hostil hacia el equipo de enfermería, al parecer porque lo ubicaba como responsables de las "agresiones" que fue recibiendo.

Finalmente, la comunicación con la señora no alcanzó, el nivel de empatía deseable para una persona que se encuentra en una situación de enfermedad terminal. Si bien el equipo estable de enfermería mantuvo durante todo el proceso un vínculo correcto en lo instrumental y procedimiental, en lo expresivo fue más bien indiferente y frío. Sólo estaban junto a la señora el tiempo estrictamente necesario. El resto del tiempo del turno, el vínculo entre enfermería y ella fue mantenido por la presencia de la estudiante de enfermería.

\section{Datos de ensañamiento terapéutico en la usuaria}

En este difícil equilibrio entre ensañamiento y desahucio prematuro cabe señalar las situaciones que obligaban a procedimientos terapéuticos inútiles. Dado que fue infructuoso el destete del ventilador mecánico, se hizo necesario realizarle gasometría para establecer los parámetros del ventilador según sus requerimientos, habiéndose indicado dos gasometrías por turno. Diariamente y durante 45 días desde su ingreso, la usuaria fue puncionada seis veces al día sólo para la regulación de los parámetros ventilatorios; un total de 270 veces.

Desde su ingreso a la Unidad de Cuidados Intensivos, se le indicó una radiografía de torax diaria que se realizaba en el turno matutino. El objetivo era monitorear la evolución del derrame pleural. Este procedimiento implicó la movilización de la señora por parte de los técnicos radiólogos, el contacto con la placa (el período corresponde al otoño - invierno), sin beneficios visibles para la señora.

En la bibliografía no existe consenso respecto a si otros procedimientos resultarían o no desproporcionados, pero pudieran ser cuestionables por ejemplo, la realización de diálisis, la manutención de un gasto cardíaco a expresas de la utilización de inotrópicos y digitálicos en un corazón que "ya no da más", la utilización de antibioticoterapia. Y finalmente cuando se produce el paro cardio respiratorio la realización de maniobras reanimatorias.
Es sabido que en los estadios finales de la sepsis, la plaquetopenia lleva a sangrados frecuentes, ya que frente a un bajo gasto cardíaco el organismo trata de suministrar oxigeno a cerebro corazón y riñones en detrimento del resto del organismo, lo cual también lleva a la producción de sangrados. Por lo tanto el procedimiento de tacto vaginal, es el indicador más claro de que hubo ensañamiento terapéutico, éste no iba a cambiar radicalmente la terapéutica aplicada ni aportar datos nuevos que condujeran a cambios en la misma.

Si se visualiza objetivamente todos estos procedimientos, se puede observar que eran desproporcionadamente molestos, e incrementaron el dolor y el sufrimiento en comparación con el resultado que se esperaba de ellos.

\section{CONCLUSIONES}

¿Tuvo la señora una muerte digna? Partiendo de la postura que establece que una muerte digna requiere brindar al moribundo todos los alivios médicos adecuados, que permitan disminuir el dolor sin que lleguen a ser desproporcionados, y además otorgarle todos los consuelos humanos posibles., se debe concluir que la señora careció de una muerte digna en ambos planos.

Sin embargo refiriéndose al segundo aspecto, los consuelos humanos otorgados, por nuestra parte como profesionales de Enfermerìa, nuestro objetivo era el de palear el dolor físico y brindarle confort e higiene en todo momento. Haciendo inclusión a la dimensión espiritual de la muerte. El proceso de morir va mucho mas allá de lo físico, teniendo connotaciones de carácter cultural, psicológicas y también ético-religiosas; máxime en una sociedad como la mexicana donde la religiosidad es un asunto vital y cotidiano.

No fue posible tampoco re-significar los procedimientos invasivos de los cuales era objeto la señora, al punto que ella los interpretó como un "pago desproporcionado" por sus culpas.

La señora profesaba la fe católica, pero careció de una atención espiritual expresa (por ejemplo, no recibió la unción de los enfermos). Si bien ingresaba un sacerdote a la Unidad de Cuidados Intensivos Neurológicos, el cual realizaba una oración y esparcía "agua bendita" a los enfermos carecía del carisma necesario para brindar consuelo y esperanza, necesarios para la lograr la paz espiritual. Considerando la posibilidad de una asistencia individualizada por parte del sacerdote. 
¿Se puede hablar de ensañamiento terapéutico? Desde el punto de vista clínico, la señora se encontraba en una situación de enfermedad terminal (SET) puesto que la evolución natural de su enfermedad crónica llegó a su fin luego de 60 años. No existían tratamientos médicos reales capaces de curarla. Para un potencial trasplante de órganos, se considera a la edad avanzada como uno de los criterios de exclusión.

Sin embargo, esta línea de razonamiento es potencialmente peligrosa. La dificultad mayor que enfrenta en términos prácticos, es la de establecer un pronóstico de vida y en consecuencia no arrastrar a un equipo de salud a una situación de "desahucio prematuro". Si bien existen una serie de instrumentos que intentan predecir el pronóstico a corto plazo de pacientes críticamente enfermos hospitalizados en la UCl (como el APACHE III Acute Physiology And Chronic Health Evaluation) sistema que permite cuantificar la gravedad de la enfermedad a través de la valoración de 34 variables fisiológicas, que expresan la intensidad de la enfermedad y, por tanto, el estado clínico del paciente.

El caso aquí presentado representó un desafío a la reflexión bio-ética, debido a su avanzada edad, a la gravedad de la persona, a su condición terminal, los dilemas planteados respecto a la utilidad de algunos procedimientos, el comportamiento del equipo de Enfermería y al abandono familiar en que murió. Pero sería un error pensar que un caso como el que aquí se presentó resulta excepcional en México. El proceso de transición demográfica iniciado en los años setenta está repercutiendo en un incremento en la edad promedio de la población usuaria de los servicios de salud, en la prevalencia de las enfermedades crónicodegenerativas, y en el incremento de la proporción de adultos mayores que fallecen hospitalizados, más exactamente en unidades de cuidados intensivos. Este nuevo perfil epidemiológico y social debe ser motivo de reflexión en la disciplina de la enfermería.

En el centro de estos problemas está el sentido que debemos dar a los cuidados de enfermería y a la relación enfermera-paciente en una situación de enfermedad terminal (SET). Hoy cuando los valores están cambiando en los individuos, donde la ecuación costo/beneficio parece primar en la elección de un tratamiento, cuando la ciencia y la técnica marcan prácticamente todos los espacios y ritmos de la atención de pacientes críticos, corremos el riesgo de perder nuestra sensibilidad humana, y de sentir afecto por la persona que está sufriendo. La máquina podrá monitorear los signos vitales del usuario con total precisión pero no podrá aliviar su angustia, acompañar el dolor, conversar sobre el sentido de lo que se está viviendo o proporcionar un ambiente más humano en los últimos momentos de la vida.

Las anteriores reflexiones tienen una especial significación para pensar muy en particular sobre el papel de la enfermera especialista en cuidados críticos del adulto en el contexto las grandes transformaciones que están ocurriendo principalmente en el campo de la tecnología aplicada a la salud. En nuestra opinión, estos cambios hacen imprescindible profundizar la sustentación de las prácticas de Enfermería en teorías y modelos apropiados. En otro lugar sostenía que el modelo de Virginia Henderson tiene la virtud de establecer explícitamente que la base de la atención de salud está en las necesidades humanas de la persona. La satisfacción de estas necesidades en el contexto de una unidad de cuidados intensivos, son las que deberían impulsar el desarrollo permanente de las habilidades, actitudes y aptitudes que la enfermera especialista necesita para proporcionar atención holística, curativa y de calidad que redunde en resultados óptimos para el paciente.

Del análisis del caso quedan planteadas dos interrogantes ¿cómo abordar el cuidado de los cuidadores? Y icómo se establece el discernimiento sobre aspectos tan complejos como el ensañamiento terapéutico si algunas opiniones profesionales no son consideradas por razones de status?

\section{REFERENCIAS BIBLIOGRÁFICAS}

1 Botella,J. Errando, E. \& Martínez, V. (2000). Los ancianos con enfermedades en fase terminal. En Eulalia López Imedio (comp) (2000) Enfermería en cuidados paliativos. Editorial Médica Panamericana. Madrid.

2 Lille FR (2003). Breve Historia de la Conquista del Dolor en México. Gaceta Mexicana del Dolor 2003; 1(3):7 y 2004; 2(1):4

3 Saunders CM. (1980). La filosofía del cuidado terminal. En: Saunders CM. Cuidados de la enfermedad maligna terminal. Barcelona: Salvat Editores.

4 Niño, L. (1994) Eutanasia: morir con dignidad. Consecuencias jurídico-penales.. Editorial Universidad. Buenos Aires.

5 Baudouin, J.L. y Blondeau D. (1995) La ética de la muerte y el derecho a morir. Editorial Herder. Barcelona.

6 Pascual, S. (2000) Aspectos éticos en cuidados paliativos. En Eulalia López Imedio (comp) (2000) Enfermería en cuidados paliativos. Editorial Médica Panamericana. Madrid.

\section{DIRECCIÓN DE CORRESPONDENCIA}

Mtra. Teresa Salazar Gómez: tsalazar98@yahoo.com.mx 